Conference Proceedings

Journal of Technology in Counselor Education and Supervision (ISSN 2692-4192)

Volume 1(1): 49 - 52

(C) 2021Journal of Technology in Counselor Education and Supervision

https://doi.org/10.22371/tces/009

\title{
Effects of Clinical Skills Training: Technology and Pedagogy for the Quality Virtual Classroom
}

\author{
Szu-Yu Chen', Kelly Coker², Cristen Wathen ${ }^{3}$, \& Donna Sheperis ${ }^{4}$
}

\begin{abstract}
There is a dearth of research on the effects of online delivery of clinical skills training. This pilot study examined the effects and experiences of 27 master's students receiving 8-week remote basic counseling skills courses. Results suggested that by the end of online training, all participants met the course criteria of skills development. There is a statistically significant increase in students' beliefs about their own capabilities to effectively facilitate counseling techniques and counseling-related activities. Four themes emerged in participants' perception of online skills training, including concerns, challenges, technology tools, and overall learning experience. Limitations and recommendations for implications and future research are discussed.
\end{abstract}

\section{Keywords}

counseling skills, online clinical training, distance counselor education

To date, the research regarding online delivery of clinical skills training remains limited while the practice does not. Particularly, a review of the literature revealed no studies examining the efficacy of online synchronized counseling skills training. Thus, more research is needed to explore counselor educators' understanding and experiences in facilitating online clinical training, as well as students' perspectives in receiving online clinical training and supervision (Chen et al., 2020). Based on this knowledge, we engaged in a pilot study to examine the effect of online clinical skills training when COVID-19 required in-person classes to move virtually. This article aims to share a distinct online pedagogy for facilitating synchronized and asynchronized clinical skills training and to provide recommendations for enhancing and assessing students' clinical skills competencies in a virtual classroom based on the study results.

\section{Course Structure}

The asynchronous components of the online Counseling Skills course included 8 weeks of course readings, recorded faculty lectures, skill demonstration videos,

\begin{tabular}{ll}
\hline 1 & Palo Alto University, Palo Alto, CA, USA \\
2 & Palo Alto University, Palo Alto, CA, USA \\
3 & Palo Alto University, Palo Alto, CA, USA \\
4 & Palo Alto University, Palo Alto, CA, USA
\end{tabular}

and weekly reflection journals through CANVAS, their learning management system. Throughout the course, we incorporated modules of Theravue, an interactive skills practice software program that allows students to watch a variety of clients and their presenting problems, and then record a counseling response to send to their instructor for feedback.

Synchronously, 15 students per class met for two hours daily in Zoom with their instructor and a teaching assistant. During class time, the instructor and teaching assistant (TA) provided skill demonstrations, led large fishbowl role plays, and divided students into triadic skills practice groups utilizing breakout rooms. Instructors and TAs were then able to provide specific individual feedback. Students met outside of class to record the baseline and final videos demonstrating counseling skills. The videos were 20 and 30 minutes, respectively, and students were asked to transcribe portions of their sessions and identify the skills used in their interventions as well as provide alternative responses as needed. These videos and transcripts were submitted to Tevera, an online clinical skills management platform, where the students and instructors also filled out the CCS-R (Lambie et al., 2018) evaluation. Storing

Corresponding Author: Szu-Yu Chen, PhD, Palo Alto University, 1791 Arastradero Road, Palo Alto, CA 94304 email: dchen@paloaltou.edu 
the information here begins the process of tracking their clinical skills as they progress towards practicum.

\section{Effects of Online Skills Training}

The purpose of this study was to investigate the effects and experiences of students receiving 8-week online counseling skills training. The research questions for this study were: (a) What are the effects of online counseling skills training on students counseling self-efficacy as measured by Counselor Activity Self-Efficacy Scales? (b) What are the effects of online counseling skills training on students counseling competencies as measured by Counselor Competencies Scale-Revised? (c) What are students' perceptions of receiving online counseling skills training using different online training tools?

\section{Participants}

The sample for this study consisted of 27 master's level counseling students who enrolled in basic counseling skills courses in a university in the Western region of the United States. See Table 1 for participant demographics.

\section{Table 1}

Participants Demorgaphic Characteristics

\begin{tabular}{lcc}
\hline Gender & $\mathrm{N}$ & $\%$ \\
Cisgender woman & 25 & 92.5 \\
Cisgender man & 1 & 3.7 \\
Prefer not to answer & 1 & 3.7 \\
Race/Ethnicity & & \\
White & 11 & 40.7 \\
Hispanic, Latinx, or Spanish of origin & 4 & 14.8 \\
Asian & 4 & 14.8 \\
Black & 2 & 7.4 \\
Middle Easter or North African & 2 & 7.4 \\
Multiracial & 4 & 14.8 \\
Age & & \\
$21-29$ & 7 & 25.9 \\
$30-39$ & 7 & 25.9 \\
$40-49$ & 8 & 29.6 \\
$50-59$ & 5 & 18.5 \\
\hline
\end{tabular}

\section{Instrument}

The Counselor Activity Self-Efficacy Scales. The Counselor Activity Self-Efficacy Scales (CASES; Lent et al., 2003) aims to assess counselors' beliefs about their ability to demonstrate a variety of counselor techniques and behaviors and to cope with particular situations in the counseling process. The CASES scale includes a total of 41 items, and three subdomains of helping skill self-efficacy, session management self-efficacy, and counseling challenges self-efficacy. Each item is presented with a likert-scale response set with values ranging from no confidence ( 0 ) to complete confidence (9). Possible scores ranged from 0 to 369, with higher scores indicating higher counselor self-efficacy beliefs. Reliability analysis of the CASES revealed an overall alpha score of .97 with subdomains ranging from .79 to .92 from 345 counseling students (Lent et al., 2003). A test-retest reliability showed .80 to .96 correlation at a two-week interval.

Counselor Competencies Scale-Revised. Counselor Competencies Scale-Revised (CCS-R; Lambie et al., 2018) was developed to assess counselors' skills development and professional competencies. The 23 item CCS-R provides counselors with direct feedback regarding their demonstrated ability to apply counseling skills and facilitate therapeutic conditions, and their counseling dispositions (dominant qualities) and behaviors, offering the counselors practical areas for improvement to support their development as effective and ethical professional counselors.

The first part of CCS-R is counseling skills and therapeutic conditions, containing 11 items and focusing on primary counseling skills and conditions that facilitate a therapeutic relationship with the client. The second part of CCS-R is counseling dispositions and behaviors, including 12 items and focusing on qualities and behaviors in counselors-in-training that are crucial for counseling competency. Each item is presented with a scale response set with values ranging from 1 (harmful) to 5 (exceeds expectations / demonstrates competencies). Possible scores ranged from 23 to 115, with higher scores indicating higher counseling competency. The interrater reliability analysis results were acceptable (counseling skills and therapeutic conditions,. 91 ; counseling dispositions and behaviors,. 56 ; total CCS$\mathrm{R}$ score,.84). The internal consistency reliability analysis results for the CCS-R were strong (counseling skills and therapeutic conditions,. 94 ; counseling dispositions and behaviors,.94; total CCS-R score,.96).

\section{Research Design}

In the current study, we employed a mixed-methods approach to gain an understanding of counseling students' online skills learning experience, counselor self-efficacy, and counseling competencies outcomes. Aligned with this methodological approach, we analyzed quantitative results of Counselor Activity Self-Efficacy Scales (Lent et al., 2003) and Counselor Competencies Scale-Revised (Lambie et al., 2018) as well 
as individual qualitative data to gain knowledge of students online clinical training experiences and perceived counseling self-efficacy (Creswell, 2015).

\section{Results}

\section{Counseling Self-Efficacy}

We hypothesized that facilitating remote counseling skills training would enhance students' overall counseling self-efficacy. A paired-samples t-test was conducted to evaluate the impact of the online counseling skills training on students' scores on the CASES. There was a statistically significant increase in the helping skill domain from the first week of the class $(M=4.92$, $S D=5.47)$ to the last week of the class $(M=6.43$, $\mathrm{SD}=3.28), \mathrm{t}(404)=-15.06, \mathrm{p}<.001$ (two-tailed). The $95 \%$ confidence interval for the effect of online skills training on helping skills self-efficacy is between 1.31 and 1.71 . The eta squared statistic (.75) indicated a large effect size.

There was a statistically significant increase in the session management subscale scores from week 1 $(M=4.66, S D=4.51)$ to week $8(M=6.44, S D=1.96), t$ $(269)=-14.37, p<.001$ (two-tailed). The magnitude of the differences in the means $(95 \% \mathrm{Cl}: 1.54$ to 2.02$)$ was large (eta squared $=.87$ ). There was a statistically significant difference in the counseling challenges self-efficacy scores for week $1(M=4.81, S D=5.63)$ and week $8(\mathrm{M}=5.76, \mathrm{SD}=4.91), \mathrm{t}(431)=-9.39, \quad \mathrm{p}<.005$ (two-tailed). The 95\% confidence interval for the effect of online skills training on challenges self-efficacy is between .75 and 1.15 . The eta squared statistic (.45) indicated a median effect size. These results suggest that online counseling skills training enhances students' beliefs about their own capabilities to effectively perform counseling techniques and counseling-related activities.

\section{Counseling Skills and Professional Competencies}

To evaluate students' skills development and professional competencies, instructors completed CCS-R in the final week. Students need to demonstrate a score of 3 (near expectations/developing towards competencies) or above to receive a passing score from the course. The results of counseling skills and therapeutic conditions $(M=3.62, S D=1.53)$ indicated that instructors perceived students' demonstration of primary counseling skills and conditions that facilitate therapeutic relationships with clients meeting the course requirements. The mean score of $3.62(S D=1.88)$ for counseling dispositions and behavior revealed that students' qualities and professional behaviors also met the course standards.

\section{Experience of Online Training}

A key part of the study was to identify perceptions of participating students. Specifically, five open-ended questions were asked in the survey to capture perceptions of the following: (a) Concerns about completing Counseling Skills in an online format, (b) Challenges experienced during the course, (c) The usefulness of the virtual counseling skills software, Theravue, (d) Overall experiencing using online tools (Zoom, Canvas, Tevera, Theravue), and (e) The Overall experience of participating in Counseling Skills virtually.

Concerns. In anticipation of participating in a virtual Counseling Skills class, students expressed concerns about the amount of time that would be available to practice skills and to get feedback on those skills. Another key concern raised was whether the technology used would provide opportunities to learn and practice non-verbal communication skills, whether technical difficulties would impede learning, and whether the skills learned could be replicated to in-person experiences.

Challenges. Actual challenges experienced during the virtual intensive week clustered around three main themes: Time management (i.e., family commitments, job commitments, other courses taken, not enough time, generally); technology issues (i.e., Zoom "freezing", lagging internet, audio and video issues); and personal toll (i.e., mentally and physically taxing, feeling overwhelmed).

Technology Tools. Two questions focused on the technology tools and platforms used for the Counseling Skills class. For the use of Canvas (LMS) and Zoom, students generally felt comfortable as they were already familiar with them from other courses. The skills practice tool, Theravue, was new to students and was generally well received as a way to augment and enhance other skills practice. Tevera, the system used for sharing videos and completing CCS-R assessments, received mixed reviews from being "confusing and tedious" to "convenient and helpful".

Overall Learning Experience. Overall, students indicated they felt supported by faculty and peers during the virtual Counseling Skills course. While concerns and issues with time was a common factor in the responses, students indicated that they learned more than they expected in the virtual format and generally felt prepared to move to the next stage of their training.

\section{Discussion and Implications}

Results showed that students improved their 
self-efficacy regarding counseling skills, succeeded in improving abilities in counseling competencies as measured by the CCS-R, and believed that in the circumstances they were under, that the platforms utilized in the course were effective for their learning. Implications derived from the study include that well planned online counseling skills synchronous classes allow counselors-in-training to develop their counseling skills, to feel confident that they are doing so, and to demonstrate that they are capable of meeting the standards of the class.

After reviewing feedback from the qualitative results, we incorporated strategies to further improve course delivery. These included extending the triadic skills practice hours by adding 30 minutes to the overall course time, utilizing most of the synchronous class meeting time for practice and processing, and incorporating a telemental health framework into the class.

\section{Limitations and Future Research}

The current research is limited by being conducted within one university environment. While students participated from across and outside of the United States, results cannot be generalized. Due to the paucity of research related to synchronous online skills training, future research could focus on effective pedagogy that enhances student learning and allows for the development of clinical competency. In addition, it is suggested that such research address the need for culturally responsive clinical practice within a synchronous, online, training environment.

\section{References}

Chen, S-Y., Wathen, C., \& Speciale, M. (2020). Online clinical training in the virtual remote environment: Challenges, opportunities, and solutions. The Professional Counselor, 10(1), 78-91. https://doi.org/10.15241/syc.10.1.78

Creswell, J. W. (2015). A concise introduction to mixed methods research. Sage.

Lambie, G., Mullen, P., Swank, J. \& Blount, A. (2018). The counseling competencies scale: Validation and refinement. Measurement and Evaluation in Counseling and Development, 51, 1-15. https://doi.org/10.1080/074817 56.2017.1358964.

Lent, R. W., Hill, C. E., \& Hoffman, M. A. (2003). Development and validation of the counselor activity self-efficacy scales. Journal of Counseling Psychology, 1, 97-108. https://doi.org/10.1037/0022-0167.50.1.97 